\title{
Arztberichte im Asylverfahren
}

Thomas Maier

Korrespondenz:

Dr. med. Thomas Maier

Ambulatorium für Folter-

und Kriegsopfer SRK

Psychiatrische Poliklinik

des Universitätsspitals Zürich

Culmannstrasse 8

CH-8091 Zürich

Tel. 0442555280

Fax 0442558645

thomas.maier@usz.ch

\section{Zusammenfassung}

Im Asylverfahren werden in zunehmendem Masse medizinische (insbesondere psychiatrische) Beurteilungen der Gesuchsteller veranlasst. In der Regel werden diese Beurteilungen in Form von Arztberichten vorgenommen, die mittels eines Standardformulars zuhanden des zuständigen Bundesamtes für Migration zu senden sind. Die herrschende Asylpraxis berücksichtigt allerdings medizinische Faktoren für die Anerkennung als Flüchtling kaum; lediglich für die Gewährung der vorläufigen Aufnahme nach ablehnendem Asylentscheid kommt der gesundheitlichen Situation des Gesuchstellers eine gewisse Bedeutung zu. Vielen Asylsuchenden, aber auch vielen Ärzten sind diese Tatsachen zu wenig bewusst. Es werden inhaltliche und formale Empfehlungen für das Abfassen von Arztberichten im Asylverfahren gegeben.

\section{Einleitung}

Auch wenn das Asylwesen infolge des Rückgangs neuer Gesuche in letzter Zeit etwas aus dem Fokus der öffentlichen Aufmerksamkeit gewichen ist, bewegt das Thema nach wie vor viele Gemüter. Ähnlich wie bei anderen sozialpolitischen Problemen ist auch im Asylverfahren ein gewisses Mass an Medikalisierung zu beobachten, das heisst, ärztlichen Beurteilungen kommt im Rahmen der Entscheidungsfindung wachsende Bedeutung zu. Konkret geschieht dies hauptsächlich durch das Erstellen von ärztlichen Berichten zuhanden des Bundesamtes für Migration (BFM, vormals Bundesamt für Flüchtlinge/ BFF). Die FMH hat sich zu Schwierigkeiten in diesem Zusammenhang auch an dieser Stelle verschiedentlich geäussert [1]. Weiterhin bestehen aber Unklarheiten und Meinungsverschiedenheiten über die Funktion von ärztlichen Stellungnahmen im Asylverfahren.

Das Asylverfahren ist ein juristisches Prozedere mit dem Ziel, festzustellen, ob eine Person die Eigenschaft eines Flüchtlings erfüllt oder nicht. Der Flüchtlingsbegriff ist in der Genfer Flüchtlingskonvention von 1951 international verbindlich definiert [2]. Flüchtling ist, wer in seinem Heimatland aufgrund von Rasse, Religion, Nationalität, Zugehörigkeit zu einer bestimmten sozialen Gruppe oder wegen politischer Anschauungen verfolgt wird und deshalb aus diesem Land fliehen musste. Der Flüchtlings-

\section{Rapports médicaux dans les procédures d'asile}

Lors des procédures d'asile, on exige de plus en plus souvent des expertises médicales (principalement d'ordre psychiatrique) sur les requérants. En règle générale, il s'agit d'un rapport médical qui doit être transmis au moyen d'un formulaire standard à l'Office fédéral des migrations. La pratique actuelle en matière d'asile n'accorde toutefois guère d'importance aux aspects médicaux dans l'octroi du statut de réfugié. C'est uniquement après le rejet de la demande d'asile, lorsqu'une admission provisoire est prononcée, que l'état de santé du requérant prend une certaine importance. De nombreux requérants d'asile et de nombreux médecins ne sont pas au courant de ce fait. Le présent article donne des recommandations tant matérielles que formelles pour l'établissement de rapports médicaux dans les procédures d'asile.

begriff hat in der Schweiz traditionell einen hohen Stellenwert, nicht zuletzt auf dem Hintergrund der zwiespältigen Rolle des Landes während des Zweiten Weltkriegs. Es gilt gewissermassen als Landestradition und entspricht unserem Selbstverständnis als Ursprungsland des Roten Kreuzes, Verfolgten und Misshandelten Schutz und Aufnahme zu gewähren. Im Zuge grosser weltweiter Wanderungsbewegungen hat sich indessen in allen Industrieländern das Asylwesen seit Jahren stark mit Problemen der Arbeits- und Wirtschaftsmigration vermischt. Auch aufgrund des Fehlens einer eigentlichen Migrationspolitik in der Schweiz müssen sich die Asylbehörden mit einer grossen Zahl von Gesuchstellern beschäftigen, welche die Flüchtlingseigenschaft nicht erfüllt. Die Unterscheidung der «echten» von den «unechten» Flüchtlingen erfordert einen hohen personellen, finanziellen, verfahrenstechnischen und intellektuellen Aufwand, der dennoch nie eine Garantie für die Korrektheit dieser Unterscheidung bietet. Ähnlich wie in anderen vergleichbaren Unterscheidungsverfahren (z. B. Invalidität, Unfallkausalität, Zu- 


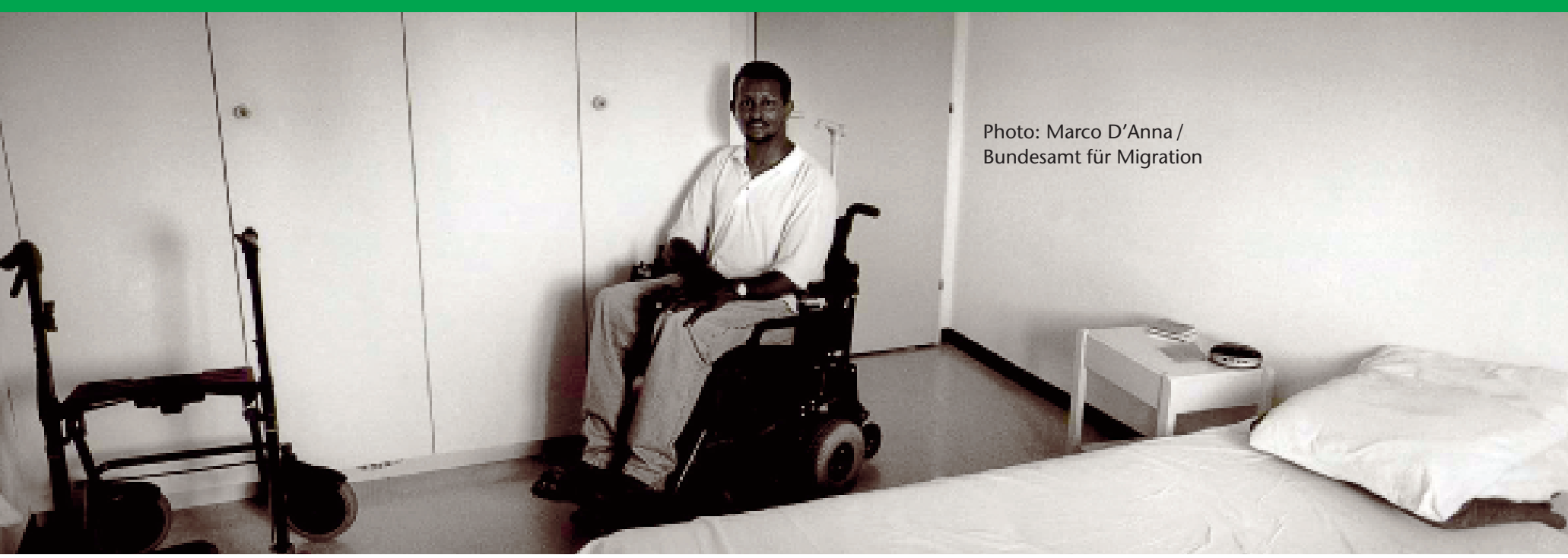

rechnungsfähigkeit) ist klar, dass selbst eine vermeintlich objektive juristische Feststellung bestimmter Sachverhalte tatsächlich nie frei von Einflüssen politischer, gesellschaftlicher und individueller Meinungen sein kann. Wie häufig an solchen heiklen Schnittstellen von Recht, Politik und Gesellschaft [3] wird auch im Falle des Asylwesens zunehmend die Medizin involviert, der die Verfahrensbeteiligten offenbar zutrauen, komplexe Sachverhalte objektiv feststellen zu können und damit den Entscheidungen höhere Legitimität zu verleihen. Der grösste Teil solcher Aufgaben fällt in der Regel in das Fachgebiet der Psychiatrie. Das BFM beobachtet seit 1998 eine stete Zunahme von Fällen, in denen medizinische Probleme geltend gemacht werden, davon betreffen $65 \%$ psychiatrische Krankheitsbilder.

\section{Asylverfahren und Medizin}

Bei der Frage, an welchem Punkt und zu welchem Zweck die Medizin in das Asylverfahren involviert werden soll und welche Bedeutung medizinischen Berichten zukommt, tauchen Unklarheiten und Meinungsverschiedenheiten auf [1]. Im Gegensatz etwa zur Invaliditätsabklärung spielen eigentliche Gutachten, d.h. ausführliche ärztliche Expertisen zu konkreten Fragestellungen im Asylwesen, quantitativ keine grosse Rolle. Neben verschiedenen anderen Gründen ist sicher auch die geringe wirtschaftliche Potenz der in Frage stehenden Klientel dafür verantwortlich. De facto werden im Asylverfahren hauptsächlich Arztberichte erstellt. Das bedeutet, dass die berichtenden Ärzte in der Regel zugleich Behandler der zu beurteilenden Person sind und zu dieser in einem Vertrauensverhältnis stehen. Die mitgeteilten Befunde sind im Rahmen von Untersuchungs- und Therapiesitzungen erhoben worden und nicht - wie es bei einer Begutachtung der Fall wäre - von einem aussenstehenden Gutachter eigens für diesen Zweck. Arztberichte sind aus diesem Grund immer in gewissem Masse parteilich, ohne deswegen fehlerhaft oder fachlich unzureichend sein zu müssen.

Das Bundesamt für Migration BFM fordert Asylsuchende, die gesundheitliche Probleme geltend machen, auf, ärztliche Berichte einzuholen. Arztberichte können auf jeder Stufe des Verfahrens eingereicht bzw. angefordert werden. Auf Anfrage bestätigt das BFM, dass die Bezahlung von Arztberichten vom Bundesamt übernommen wird, wenn diese auf Aufforderung der Behörden erstellt werden. Wenn Asylsuchende auf eigene Initiative Arztberichte verfassen lassen, gehen diese hingegen zu deren Lasten. Seit langem verfügt das BFM über ein Formular «Ärztlicher Bericht», auf dem ähnlich wie bei IV-Arztberichten Angaben zur Anamnese, zum Gesundheitszustand und zur nötigen Therapie gemacht werden können. Das Formular ist in elektronischer Form verfügbar (www.bfm.admin.ch) und wurde in der neuesten Version leicht verändert. (Die umstrittene Frage nach der Reisefähigkeit wurde - zu Recht - ersatzlos gestrichen.)

Was sollen Ärzte zuhanden des BFM attestieren? Je nach Standpunkt und Interessenlage erwarten Asylbewerber, deren Rechtsvertreter, die behandelnden Ärzte und die Behören offensichtlich je Unterschiedliches. Der Arztbericht im Asylverfahren ist daher immer in Gefahr, für die Interessen der einen oder anderen Seite instrumentalisiert zu werden. Wie immer in solchen Situationen kann die Konsequenz daraus nur darin bestehen, die Arztberichte fachlich möglichst fundiert, transparent und nachvollziehbar zu verfassen.

Oft herrscht bei Asylsuchenden (aber auch bei Ärzten) die irrige Annahme, dass der Gesundheitszustand der asylsuchenden Person im Hinblick auf eine Anerkennung als Flüchtling eine Rolle spiele. Tatsächlich wird in der herrschenden Asylpraxis einzig beurteilt, ob eine Person im Her- 
kunftsland verfolgt wird oder nicht. Erst im Falle einer Ablehnung des Asylgesuchs kommt dem aktuellen Gesundheitszustand Bedeutung zu. Dies, weil die erst an diesem Punkt des Verfahrens mögliche Gewährung einer vorläufigen Aufnahme (Aufenthaltsstatus F) unter anderem aufgrund des Vorliegens einer im Herkunftsland nicht adäquat behandelbaren Krankheit erfolgen kann. Das BFM berücksichtigt also gesundheitliche Probleme primär im Hinblick auf die Zumutbarkeit und Zulässigkeit der Rückkehr bzw. Rückschaffung in das Herkunftsland. Gemäss Rechtspraxis erfolgt eine vorläufige Aufnahme aus medizinischen Gründen, wenn «kurze Zeit nach der Rückkehr eine wesentliche, unter Umständen lebensbedrohliche Verschlechterung des Gesundheitszustandes zu befürchten ist, weil die ausreichende medizinische Behandlungsmöglichkeit vor Ort fehlt». Die hauptsächliche Funktion des Arztberichts besteht also darin, für den Fall der Ablehnung des Asylgesuchs den Behörden Grundlagen zu liefern, diese Frage zu beurteilen. Insofern benötigen die Asylbehörden eher Angaben zum aktuellen Gesundheitszustand und zu vorliegenden Krankheiten als zur Anamnese. Beim Vorliegen bestimmter Krankheiten benötigt das BFM möglichst genaue Angaben darüber, wie die Krankheit behandelt werden muss, um abzuschätzen, ob eine Behandlung im Herkunftsland möglich ist oder nicht. Bei der Entscheidungsfindung in diesen Fällen orientiert sich das BFM laut eigenen Angaben seit Juni 2004 an einer intern festgelegten Amtspraxis, in welcher die Zumutbarkeitskriterien genauer definiert sind.

Eine minutiöse Dokumentation von Folterspuren und -folgen, wie dies im internationalen Kontext der Menschenrechtsbewegung empfohlen und gefordert wird [4], sehen die Asylbehörden unter Umständen nicht für relevant an, da daraus nicht automatisch eine aktuelle Bedrohung abgeleitet wird. Man mag diese Argumentation für diskutabel oder gar für indiskutabel halten, sie gibt einfach die - letztlich politisch abgesegnete - Rechtspraxis wieder.

Besonders im psychiatrischen Bereich stellt sich oft das Problem, dass Störungen diagnostiziert werden, deren Behandlung gerade durch die spezifischen Lebensbedingungen als Asylbewerber - unsicherer Aufenthaltsstatus, laufendes Verfahren, Arbeits- und Ausbildungsverbot usw. erschwert wird bzw. deren wirksame Behandlung weniger in bestimmten medizinischen Massnahmen als in der Zuerkennung eines gesicherten Aufenthalts bestehen würde. Auf dieses Dilemma weisen wir in Arztberichten hin, allerdings im Wissen, dass dies für die Asylbehörden kein anschlussfähiges Argument ist.
Mehrfach wurde kritisiert bzw. bezweifelt [1], ob das BFM fachlich in der Lage ist, eingereichte Arztberichte zu beurteilen und angemessen $\mathrm{zu}$ würdigen. Es wird gefordert, dass ein vertrauensärztlicher Dienst zur Verfügung stehen soll, welcher Objektivität und Fachkompetenz sichert. Das BFM erklärt auf Anfrage dazu, über eine medizinische Fachstelle zu verfügen und mit Hilfe interner Fachpersonen und externer Spezialisten über die entsprechende Fachkompetenz zu verfügen. Über die Verhältnisse bei der Asylrekurskommission (ARK) ist uns nichts bekannt.

\section{Glaubwürdigkeit}

Ein umstrittenes Thema im Zusammenhang mit Arztberichten im Asylverfahren ist die Glaubwürdigkeitsbeurteilung. Für die Asylbehörden (d.h. BFM und ARK) dreht sich ein grosser Teil des Verfahrens um die Frage der Glaubwürdigkeit der Aussagen. Die eingangs erwähnte Unterscheidung von «echten» und «unechten» Flüchtlingen erfordert es, ähnlich wie in Strafverfahren, Aussagen im Hinblick auf ihre Konsistenz und Glaubwürdigkeit zu bewerten, insbesondere weil meist wenig andere objektive Beweise vorliegen. Das BFM konzentriert sich im Rahmen seines Auftrags im Asylverfahren darauf, Hinweise für fehlende Glaubwürdigkeit von Aussagen zu finden. Auch die eingereichten Arztberichte werden unter anderem auf diesen Aspekt hin geprüft. Mit umgekehrten Vorzeichen erwarten allerdings auch Gesuchsteller selbst sowie deren Rechtsvertreter von Arztberichten Unterstützung ihrer Sache, d.h. «Beweise» oder zumindest Indizien für die Richtigkeit der vorgebrachten Angaben. In diesem Spannungsfeld müssen sich die berichtenden Ärzte klar werden, wie und worüber sie sich äussern sollen und können.

Aus Verfahrensentscheiden geht hervor, dass das BFM die Ansicht vertritt, Arztberichte könnten nicht die Funktion erfüllen, einem Gesuchsteller in einem juristischen Sinne Glaubwürdigkeit zu attestieren. Wir teilen diese Meinung. Die Beurteilung der Glaubwürdigkeit von Aussagen gilt allgemein als ein schwieriges forensischpsychiatrisches Spezialgebiet, das höchste Anforderungen an Erfahrung, Methodik und Sorgfalt stellt [5-7]. Solche Fragen sind nach heutiger Lehrmeinung nur durch die Anwendung spezieller Techniken und Methoden der Aussagenpsychologie zu beantworten. In Asylverfahren liegen zudem meist sehr komplexe politischhistorisch-kulturelle Umstände vor, die eine solche Abklärung zusätzlich erschweren. Dazu kommt in aller Regel noch das Sprach- bzw. Übersetzungsproblem. Es ist somit in der Tat fraglich, ob im Rahmen eines einfachen ärzt- 
lichen Berichts Nichtforensiker überhaupt Aussagen zur Glaubwürdigkeit von Asylvorbringen machen können bzw. sollen. Arztberichte, die entsprechende Aussagen machen, setzen sich rasch dem Vorwurf aus, unprofessionell oder parteilich zu sein; das BFM kritisiert nicht zu Unrecht diesbezüglich zu wenig fundierte Berichte. Kuhn und Steiner-König [1] schlagen dennoch vor, in Anlehnung an die im Istanbul-Protocol [4] gegebenen Definitionen medizinische Konsistenzbeurteilungen zuhanden des BFM vorzunehmen. Es sei international eine Selbstverständlichkeit, dass Ärzte im Zusammenhang mit möglicher Folter Stellung nähmen. Dabei bewegt sich der Arzt aber auf einem schmalen Grat und muss sich verschiedener Probleme bewusst sein:

Als behandelnde Ärzte nehmen wir gegenüber unseren Patienten notwendigerweise eine empathische, solidarische Haltung ein und gewähren einen Vertrauensvorschuss. Dies gilt erst recht, wenn schwere Traumatisierungen zu vermuten sind. Da wir im Rahmen einer effektiven Behandlung auf der Seite des Patienten stehen müssen, wird das Resultat einer Konsistenzprüfung praktisch immer eine hohe Glaubwürdigkeit ergeben. Dieser in guten Treuen und ohne Beschönigungsabsicht erhobene Befund kann nicht als objektives Argument im juristischen Sinn verkauft werden und wird von seiten der Asylbehörden auch kritisch beurteilt. Es kann von einem behandelnden Arzt nicht erwartet werden, dass er seinem Patienten nur mittlere oder gar geringe Glaubwürdigkeit attestieren würde. Es wäre nur unter Verleugnung der eigenen Befangenheit als Behandler möglich zu glauben, man könne juristisch objektiv etwas über die Glaubwürdigkeit von Asylvorbringen sagen. Gerade als Therapeuten müssen wir darauf achten, den Diskurs des Asylverfahrens nicht mehr als nötig mit der Therapie zu vermischen, indem wir z.B. im Hinblick auf eine Konsistenzbeurteilung im Gespräch mit den Patienten die Glaubwürdigkeit ihrer Lebensgeschichte als verhandelbaren Gegenstand behandeln.

Wir konzentrieren uns in unserer Institution darauf, zuhanden des BFM die erhobenen Befunde und die von den Patienten gemachten Angaben zur Vorgeschichte genau darzustellen. Wir attestieren, dass die Befunde mit der geschilderten Anamnese kompatibel sind, verzichten aber auf eine graduelle Abstufung dieser Feststellung.

\section{Problem PTSD}

Eine besondere Schwierigkeit ergibt sich gelegentlich bei der Diagnose der posttraumatischen Belastungsstörung (PTSD): Diese Diagnose ist entgegen der sonst strikt deskriptiven Kon- zeption der psychiatrisch-diagnostischen Manuale ICD-10 und DSM-IV kausalätiologisch definiert. Die Diagnosestellung erfordert den Nachweis eines auslösenden Traumas. Konstatiert der Arzt in seinem Bericht ein stattgefundenes Trauma, haben BFM und ARK dies in einigen Fällen als Übergriff in den eigenen Kompetenzbereich der Tatsachenfeststellung kritisiert. Die Vorbringen des Gesuchsstellers seien nämlich in casu unglaubwürdig und ergo könne auch keine entsprechende Störung als Folge davon entstanden sein. Der betreffende Arztbericht ist dadurch indirekt als Gefälligkeitszeugnis desavouiert. Dieser argumentative Zirkelschluss würde allerdings ausschliessen, dass in Arztberichten zuhanden der Asylbehörden die Diagnose PTSD überhaupt gestellt werden könnte. In Deutschland wurde dieses Problem der PTSD-Diagnose im Asylverfahren analog diskutiert. Volbert [5] erkennt, dass der Gutachter durch die Frage nach PTSD implizit $\mathrm{zu}$ einer Stellungnahme zur Glaubwürdigkeit verleitet wird. Die Autorin empfiehlt dazu lakonisch, es sei klinisch festzustellen, ob die Symptome eines PTSD vorliegen oder nicht. Es müsse klar sein, dass aus dem Vorliegen klinischer Symptome kein Rückschluss auf spezifische Auslösebedingungen möglich sei. Falls aber die Frage nach der Glaubhaftigkeit der Angaben zur individuellen Verfolgung zu beantworten sei, so müsse dies mit Hilfe aussagepsychologischer Methoden erfolgen [5]. Solche Methoden erfordern, wie erwähnt, einen hohen Spezialisierungsgrad und können nur im Rahmen aufwendiger Gutachten zur Anwendung kommen.

Falls ein PTSD vorliegt, soll dieses klar und deutlich festgestellt werden. Mit der Diagnosestellung ist automatisch ebenfalls ausgesagt, dass ein Trauma stattgefunden hat. Wie, wann und unter welchen Umständen dieses Trauma vorgefallen ist, stellt der behandelnde Arzt im Rahmen der Therapie mit für ihn genügender Sicherheit anhand des Inhalts von Intrusionen und Alpträumen problemlos fest. Dass dies für das BFM keine rechtsgenügende Beweiskraft haben kann, gehört zu den Realitäten des Asylverfahrens. Dass die posttraumatische Belastungsstörung nur einen kleinen Bereich der möglichen psychopathologischen Traumafolgen abdeckt, sei der Vollständigkeit halber hier nochmals erwähnt [4].

\section{Empfehlungen}

Arztberichte im Asylverfahren sollen u.E. das Hauptgewicht darauf legen, aktuelle Befunde möglichst präzise und fachlich korrekt zu beschreiben. Abgeleitet aus dieser syndromalen Beschreibung der Befunde können entsprechende 
Diagnosen nachvollziehbar gestellt werden. Wenn Krankheiten diagnostiziert werden, sollen Angaben über die nötige Behandlung hinzugefügt werden. Die anamnestischen Angaben sind ohne Wertung und ohne weiteren Kommentar als Aussagen des Gesuchstellers wiederzugeben. Auf spekulative Äusserungen zu kausalen Verknüpfungen soll verzichtet werden. Allenfalls muss die nicht weiter abgestufte Aussage genügen, die erhobenen Befunde seien vereinbar mit den vorgebrachten anamnestischen Angaben. Insofern decken sich diese Empfehlungen mit den Erwartungen, die das BFM an eingereichte Arztberichte hat. Die Berichte sollen laut BFM:

- verständlich sein;

- von fachlich zuständigen bzw. spezifisch kompetenten Fachpersonen verfasst werden;

- objektiv sein;

- transparent sein,

- Substanz enthalten;

- verständliche Angaben zu aktueller und notwendiger Therapie machen.

\section{Danksagung}

Ich danke Dr. phil. Julia Müller und Dr. med. Jochen Binder vom AFK Zürich für das Gegenlesen des Manuskripts. Ebenfalls danke ich lic. phil.
Patricia Ganter, Leiterin Fachgruppe Medizinalfälle, Bundesamt für Migration, für die Durchsicht dieses Textes und die wichtigen Informationen.

\section{Literatur}

1 Kuhn HP, Steiner-König U. Ärztliche Berichte und Gutachten im Asylbereich - ausgewählte Aspekte aus Sicht der FMH. Schweiz Ärztezeitung 2002; 83(36):1873-80 (ebenfalls publiziert in: Asyl 3/2002 S. 3-10)

2 UNHCR. The refugee convention. 1951. www.unhcr.ch/cgi-bin/texis/vtx/home.

3 Maier T. Das Medizinalsystem als Ort sozialer Konsensfindung. Schweiz Ärztezeitung 2001; 82(39):2065-7.

4 UNHCHR. Istanbul Protocol. 1999. www.unhchr.ch/pdf/8istprot.pdf.

5 Volbert R. Beurteilung von Aussagen über Trauma. Erinnerung und ihre psychologische Bewertung. Bern, Göttingen, Toronto, Seattle: Hans Huber; 2004.

6 Birck A. Traumatisierte Flüchtlinge - Wie glaubhaft sind ihre Aussagen? Heidelberg: Asanger; 2002.

7 Möller A, Maier P. Akzeptanz forensischer Gutachten zur Frage der Zeugenglaubhaftigkeit Daten einer explorativen Zürcher Studie. Psychiatrische Praxis 2001;28:409-10. 